\title{
Facteurs de variation non génétique de la productivité des brebis de race Noire de Thibar
}

\author{
I. Ben Salem ${ }^{1 *}$ M. Rekik ${ }^{1}$ H. Hammami ${ }^{2}$ \\ M. Ben Hamouda ${ }^{3}$ R. Aloulou ${ }^{4}$ L. Sâadoun ${ }^{2}$
}

Mots-clés

Ovin - Brebis - Taux de natalité Taille de portée - Viabilité Productivité - Tunisie.

\section{Résumé}

Afin de mesurer les performances reproductives et de productivité (poids total de la portée) de la brebis Noire de Thibar, et de déterminer l'influence de facteurs non génétiques sur ces paramètres, une base de données initiale, constituée par 101000 agneaux contrôlés pour leur croissance dans le cadre du programme national d'amélioration des ovins à viande, a été utilisée. Une base de données indexée par brebis a ensuite été générée avec un total de 64137 agnelages représentant 12 années (1991-2002), 25 fermes et 48 troupeaux. Le taux de mise bas moyen dans les troupeaux de la race Noire de Thibar a été de 80,1 $\pm 12,9$ p. 100 avec des effets importants $(p<0,001)$ de la ferme et de l'année de mise bas. A la naissance, la taille de portée moyenne par brebis mettant bas a atteint 1,32 $\pm 0,47$; elle a baissé à 1,23 $\pm 0,55$ à 70 jours après l'agnelage. Toutes les principales sources de variation retenues dans le modèle (ferme, année de mise bas, mois de mise bas et âge de la brebis), ainsi que leurs interactions ont significativement affecté la taille de la portée aux différents stades après la mise bas. La survie des agneaux par portée entre la naissance et 70 jours a été observée pour 89,9 p. 100 des brebis ; seulement 5,8 p. 100 des brebis ont perdu la totalité des agneaux de leur portée. Une analyse de la variance a révélé que les facteurs année de mise bas, mois de mise bas, âge de la brebis, sexe de la portée et taille de la portée ont eu un effet très significatif sur les proportions de brebis présentant des niveaux différents de survie des agneaux au sein de la portée. En moyenne, la productivité des brebis Noires de Thibar à 10, 30 et 70 jours après l'agnelage a été respectivement de 6,6 \pm $2,2,10,3 \pm 3,5$ et $18,6 \pm 6,6 \mathrm{~kg}$. Les principales sources de variations (ferme, année de mise bas, mois de mise bas, âge de la brebis et taille de la portée), ainsi que certaines interactions de deuxième niveau ont eu des effets significatifs $(p<0,001)$ sur les caractères de productivité étudiés. La catégorie des brebis âgées de quatre ans ayant eu des portées de triplets tous mâles a été associée aux niveaux de productivité les plus élevés à 70 jours. Ce travail préliminaire sur les caractères reproductifs et de productivité des brebis Noires de Thibar est essentiel à l'élaboration d'un index de sélection femelle qui pourra être incorporé dans un éventuel schéma d'amélioration génétique de cette race.
1. Service de Zootechnie, Ecole nationale de médecine vétérinaire, 2020 Sidi Thabet, Tunisie. 2. Direction d'amélioration génétique, Office de l'élevage et des pâturages, Sidi Thabet, Tunisie. 3. Institution de la recherche et de l'enseignement supérieur agricoles, Tunis, Tunisie. 4. Institut supérieur agronomique de Chott Meriem, Chott-Meriem, Sousse, Tunisie. * Auteur pour la correspondance

Tél. : +216 71552200 ; fax : +216 71552441 ; e-mail : bensalemimen@yahoo.fr

\section{INTRODUCTION}

Quel que soit le système de production, la productivité des brebis est la principale préoccupation des élevages ovins à travers le monde (31). Son amélioration constitue un objectif important de l'industrie de la production de viande ovine. Le nombre et le poids des agneaux sevrés par brebis ayant mis bas sont des caractères de nature composite qui sont essentiels dans la détermination de la production totale d'agneaux par brebis. Ils peuvent ainsi être considérés comme des indices phénotypiques naturels synthétisant les différents paramètres zootechniques plus élémentaires comme les taux de mise bas, la taille de la portée, la survie néonatale et le 
poids des agneaux au sevrage (17). Les voies d'amélioration de la productivité sont nombreuses. Certaines s'adressent à l'amélioration des performances reproductives (18) alors que d'autres, dans le cas des élevages à viande, ciblent les performances de croissance des agneaux (15).

Il existe une autre approche, celle d'intégrer à la fois les caractères de reproduction et la croissance dans le concept unique de la productivité par femelle reproductrice. Cette approche a donc l'avantage de considérer, en même temps, les aptitudes reproductives de la mère (prolificité), ses qualités maternelles (potentiel d'allaitement, survie des individus dans la portée), ainsi que le potentiel de croissance de ses descendants (16). Cette voie est particulièrement intéressante dans les systèmes à faibles intrants de la rive sud de la Méditerranée. En effet, dans un tel contexte, les contraintes liées à l'environnement de l'élevage des filières extensives des petits ruminants peuvent agir d'une manière continue durant tout le cycle de production des troupeaux affectant ainsi les différentes composantes de la productivité des brebis. La notion de productivité peut ainsi faire émerger des modalités d'action globales des facteurs de l'environnement, plus difficiles à décrire lorsque les composantes de la productivité sont analysées séparément.

En Tunisie, qui compte près de quatre millions de brebis (29), peu d'études ont porté sur la productivité des races autochtones au sein de leur écosystème d'élevage. A la connaissance des auteurs, la seule estimation de productivité est celle rapportée par Jmal (20) qui la situe à $20,3 \mathrm{~kg}, 90$ jours après la mise bas, pour la Barbarine à Queue grasse dans des troupeaux pilotes. Beaucoup moins importante que la Barbarine en termes d'effectifs, la race Noire de Thibar, avec environ 80000 à 90000 femelles reproductrices, est présente uniquement dans l'étage bioclimatique subhumide (32) et occupe ainsi le milieu d'élevage le plus propice en Tunisie. La race est une création du début du $\mathrm{XX}^{\mathrm{e}}$ siècle, issue d'un croisement entre la Queue fine de l'Ouest et la Mérinos de la Crau (21). Selon Chafik (12), la Noire de Thibar présente des caractéristiques zootechniques intéressantes, notamment en rapport avec sa prolificité, mais ne bénéficie pour l'instant d'aucun schéma d'amélioration.

Afin de renforcer les outils d'une évaluation génétique des brebis Noires de Thibar en se basant sur la productivité, les objectifs du présent travail ont été de quantifier les performances de reproduction et de productivité de ces brebis, et d'analyser les différentes sources de variation qui reflétaient le mode d'action du milieu sur les paramètres étudiés.

\section{MATERIEL ET METHODES}

\section{Animaux, milieu et conduite}

Dans cette étude ont été utilisées les données des brebis Noires de Thibar inscrites au contrôle officiel de croissance, conformément aux dispositions de l'International Committee of Animal Recording (6), avec absence de contrôle de la paternité. Un total de 64137 agnelages représentant 12 années (1991-2002) provenant de 48 troupeaux appartenant à 25 fermes ont été exploités. En raison de la restructuration de certaines fermes du secteur étatique au secteur privé et de l'apparition de certains foyers de maladies contagieuses (fièvre aphteuse et brucellose), le contrôle des performances a dû être suspendu et n'a pas pu se dérouler normalement durant certaines campagnes dans certaines fermes.

Les brebis inscrites au programme de contrôle de la croissance sont élevées dans des troupeaux de 200 à 250 femelles en moyenne appartenant à des fermes privées, des unités coopératives de production ou des domaines agricoles étatiques avec une conduite du type semi-intensif. Le système d'alimentation est principalement intégré à celui de la production céréalière (pâturage sur chaumes, jachères et orge en vert...). Les animaux reçoivent en plus un apport fourrager sous forme de foin, d'ensilage et de concentré en quantités variables à différents stades du cycle de production (avant la mise à la lutte, fin de la gravidité et début de la lactation).

Les troupeaux sont soumis à un rythme d'un agnelage par an avec des luttes de contre-saison s'effectuant entre les mois de mars et de juin, et des mises bas qui s'échelonnent entre les mois de juillet et de décembre. Les femelles sont mises à la reproduction pour la première fois à l'âge de 18 mois au stade antenaise. Enfin, les services de contrôle de croissance procèdent annuellement aux opérations de réforme et au choix des femelles de renouvellement avec un taux de remplacement de l'ordre de 18 p. 100 dans les troupeaux de cette étude (figure 1).

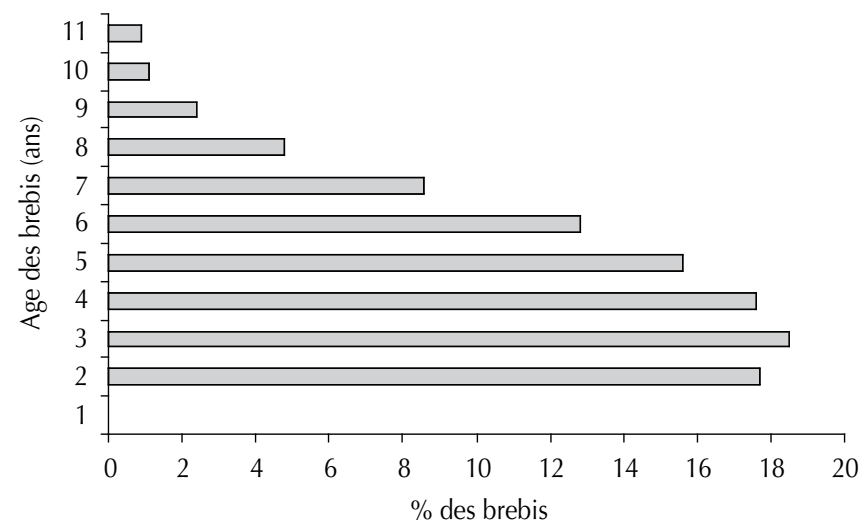

Figure 1 : pyramide d'âge des brebis dans les élevages contrôlés de la race Noire de Thibar.

\section{Paramètres étudiés}

La base de données recueillie a permis d'étudier les paramètres suivants relatifs à la productivité des brebis Noires de Thibar :

- le taux de mise bas défini par le rapport du «nombre de femelles mettant bas » sur le «nombre de femelles mises à la lutte » x 100. Le taux de mise bas a été calculé par troupeau et par an, et il a été relatif à une mise à la lutte s'échelonnant sur deux à trois mois selon les élevages ;

- la taille de la portée aux âges types, calculée en additionnant le nombre d'agneaux nés d'une même brebis et vivants à la naissance (TPN), à 30 (TP30) et à 70 (TP70) jours après la mise bas ;

- le poids de la portée aux âges types (PP10, PP30 et PP70) qui, pour une brebis donnée, représente la somme des poids individuels des agneaux issus d'une même mise bas aux âges correspondants, c'est-à-dire à 10, 30 et 70 jours après la mise bas.

Bien que le facteur sexe ne soit pas appréhendé de manière explicite dans les études de productivité pondérale, il en a été tenu compte dans ce travail eu égard à son effet mis en évidence sur les poids aux âges types et les gains moyens quotidiens individuels des agneaux $(2,5,20)$. Ainsi, pour la variable sexe de la portée (SXP) trois niveaux de variation ont été définis : les portées composées exclusivement d'individus de sexe mâle, les portées composées exclusivement d'individus de sexe femelle, et les portées composées d'individus des deux sexes.

\section{Analyses statistiques}

\section{Taux de mise bas}

L'influence des facteurs de variation du taux de mise bas dans les troupeaux de race Noire de Thibar a été étudiée à l'aide d'un 
modèle linéaire (modèle 1) qui a tenu compte des facteurs fixes suivants :

$Y_{i j k l}=\mu+F E_{i}+A M B_{j}+T R_{k}(F E)_{i}+F E x A M B_{i * j}+E_{i j k l}$

où $Y_{i j k l}$ représentait le taux de mise bas à l'échelle du troupeau appartenant à la i ferme (de 1 à 25), au cours de la je année de mise bas (de 1991 à 2002). $T R_{k}\left(F E_{i}\right)$ représentait l'effet du troupeau hiérarchisé par ferme et $F E \times A M B_{i * j}$ celui de l'interaction ferme et année de mise bas.

\section{Paramètres de productivité}

Les facteurs de variation des paramètres de productivité numérique (taille de la portée) ont été analysés avec le modèle linéaire suivant (modèle 2) :

$Y_{i j k l m}=\mu+F E_{i}+A M B_{j}+M B_{k}+A B_{l}+F E x M B_{i * k}+$ $A M B \times M B_{j * k}+E_{i j k l m}$

où $Y_{i j k l m}$ représentait la taille de la portée à la naissance, à 30 et à 70 jours après la mise bas de la brebis $\mathrm{m}$ appartenant à la $\mathrm{i}^{\mathrm{e}}$ ferme (de 1 à 25), ayant mis bas au cours de la je année de mise bas (de 1991 à 2002) et du $\mathrm{k}^{\mathrm{e}}$ mois de mise bas (entre juillet et décembre). $A B_{l}$ représentait l'effet du $\mathrm{l}^{\mathrm{e}}$ âge de la brebis (de 2 à 11 ). $F E \times M B_{i * k}$ était celui de l'interaction ferme et année de mise bas, et $A M B \times M B_{j^{*} k}$ l'effet de l'interaction année et mois de mise bas.

Enfin, les variables pondérales (poids de la portée) ont été étudiées selon le modèle 3 :

$Y_{i j k l m n o p}=\mu+F E_{i}+A M B_{j}+M B_{k}+A B_{l}+S X P_{m}+T P N_{n}+T R_{o}\left(F E_{i}\right)$

$+F E x A M B_{i * j}+A M B \times M B_{j * k}+S X P \times T P N_{m * n}+E_{i j k l m n o p}$

où $Y_{i j k l m n o p}$ représentait les poids de la portée à 10,30 et 70 jours de la brebis $\mathrm{p}$ dans la $\mathrm{i}^{\mathrm{e}}$ ferme (de 1 à 25), ayant mis bas au cours de la $\mathrm{j}^{\mathrm{e}}$ année de mise bas (de 1991 à 2002) et du $\mathrm{k}^{\mathrm{e}}$ mois de mise bas (entre juillet et décembre). $A B_{l}$ représentait l'effet du $1^{\mathrm{e}}$ âge de la brebis (de 2 à 11), $S X P_{m}$ celui du me sexe de la portée (mâle, femelle, mixte), $T P N_{n}$ l'effet de la $\mathrm{n}^{\mathrm{e}}$ taille de la portée à la naissance (simple, double, triple), $T R_{o}\left(F E_{i}\right)$ l'effet du troupeau hiérarchisé par la ferme, et $F E \times A M B_{i * j}, A M B \times M B_{j^{* k}}$ et $S X P \times T P N_{m^{*} n}$ les effets respectifs des interactions entre ferme et année de mise bas, entre année et mois de mise bas, et entre sexe et taille de la portée.

En plus de la détermination des sources de variation, les solutions des moindres carrés issues du modèle 2 et relatives aux effets fixes de l'âge de la mère, ainsi que celles relatives au modèle 3 et concernant les effets fixes du sexe de la portée, de la taille de la portée à la naissance et de l'âge de la mère ont été calculées pour décrire et quantifier l'effet intrinsèque de ces facteurs sur les tailles et les poids de la portée.

L'analyse du taux de mise bas ainsi que des paramètres de productivité numérique et pondérale a été effectuée en adoptant la procédure du modèle linéaire généralisé (GLM) du logiciel SAS (33).

\section{RESULTATS}

\section{Taux de mise bas}

Le taux moyen de mise bas des brebis Noires de Thibar a été de $80,1 \pm 12,9$ p. 100 (tableau I). Le taux de mise bas a été influencé $(\mathrm{p}<0,001)$ par les sources de variation suivantes : la ferme, l'année de mise bas et l'interaction entre ces deux facteurs. Le facteur troupeau hiérarchisé par ferme s'est révélé non significatif au seuil de $\mathrm{p}<0,05$.

\section{Taille de la portée}

Le tableau II montre les tailles de la portée moyennes à la naissance, à 30 et à 70 jours après la mise bas des brebis Noires de Thibar élevées dans le nord de la Tunisie. L'analyse statistique a mis en évidence l'influence très significative de toutes les sources de variation retenues dans le modèle (la ferme, l'année de mise bas, le mois de mise bas, l'âge de la brebis, les interactions entre la ferme et l'année de mise bas, et entre l'année et le mois de mise bas) pour les tailles de la portée à la naissance, à 30 et à 70 jours après la mise bas.

\section{Tableau I}

Sources de variation du taux de mise bas des troupeaux de race Noire de Thibar

$\begin{array}{lccccc}\text { Paramètre } & \begin{array}{c}\text { Nb. } \\ \text { d'observations }\end{array} & \text { Moy. } \pm \text { E.T. } & \mathbf{R}^{2} & \text { Effet fixe } & \text { P } \\ \begin{array}{lccccc}\text { Taux de } & 261 & 80,12 \pm 12,90 & 0,88 & \text { FE } & * * * \\ \text { mise bas (\%) } & & & & \text { AMB } & * * * \\ & & & \text { TR (FE) } & \text { NS } \\ & & & \text { FE x AMB } & * * *\end{array}\end{array}$

E.T. : écart-type ; FE : ferme ; AMB : année de mise bas ; TR : troupeau **** $\mathrm{P}<0,001$

\section{Tableau II}

Sources de variation des tailles de la portée des brebis de race Noire de Thibar

\begin{tabular}{|c|c|c|c|c|c|}
\hline Caractère & $\begin{array}{c}\text { Nb. } \\
\text { d'observations }\end{array}$ & Moy. \pm E.T. & $\mathbf{R}^{2}$ & Effet fixe & $\mathbf{P}$ \\
\hline \multirow[t]{6}{*}{ TPN } & 64137 & $1,32 \pm 0,47$ & 0,09 & $\mathrm{FE}$ & $* * *$ \\
\hline & & & & $\mathrm{AMB}$ & $* * *$ \\
\hline & & & & $\mathrm{MB}$ & $* * *$ \\
\hline & & & & $A B$ & $* * *$ \\
\hline & & & & FE $\times$ AMB & $* * *$ \\
\hline & & & & $\mathrm{AMB} \times \mathrm{MB}$ & $* * *$ \\
\hline \multirow[t]{6}{*}{ TP30 } & 61161 & $1,27 \pm 0,51$ & 0,10 & $\mathrm{FE}$ & $* * *$ \\
\hline & & & & $\mathrm{AMB}$ & $* * *$ \\
\hline & & & & $M B$ & $* * *$ \\
\hline & & & & $A B$ & $* * *$ \\
\hline & & & & FE $x$ AMB & $* * *$ \\
\hline & & & & $\mathrm{AMB} \times \mathrm{MB}$ & $* * *$ \\
\hline \multirow[t]{6}{*}{ TP70 } & 55611 & $1,23 \pm 0,55$ & 0,11 & $\mathrm{FE}$ & $* * *$ \\
\hline & & & & $\mathrm{AMB}$ & $* * *$ \\
\hline & & & & $\mathrm{MB}$ & $* * *$ \\
\hline & & & & $\mathrm{AB}$ & $* * *$ \\
\hline & & & & $\mathrm{FE} \times \mathrm{AMB}$ & $* * *$ \\
\hline & & & & $\mathrm{AMB} \times \mathrm{MB}$ & $* * *$ \\
\hline
\end{tabular}

E.T. : écart-type ; FE : ferme ; AMB : année se mise bas ; MB : mois de mise bas ; $\mathrm{AB}$ : âge de la brebis ; TPN : taille de la portée à la naissance ; TP30 et TP70 : taille de la portée respectivement à 30 et 70 jours après la mise bas *** $\mathrm{P}<0,001$ 
La figure 2 montre l'effet de l'âge de la brebis sur le nombre d'agneaux nés. Les tailles de portée des brebis dont l'âge était compris entre 5 et 6 ans ont été les plus importantes. Dans le cas de la présente étude, les brebis âgées de 5 ans ont été prises comme base de comparaison ; les résultats ont indiqué ensuite qu'elles étaient en outre celles dont les performances ont été maximales à la naissance, à 30 et à 70 jours après la mise bas. Les performances les plus faibles ont été observées chez les brebis primipares.

La TPN a été en moyenne de 1,35 en juillet, août et septembre, soit supérieure respectivement de 0,08 et de 0,23 agneaux aux TPN d'octobre et des mois cumulés de novembre et décembre (figure 3). A 70 jours après la mise bas, ces écarts ont augmenté et sont devenus respectivement de 0,10 et 0,39 agneaux.

\section{Paramètres de productivité}

Les poids de la portée à 10 , à 30 et à 70 jours après la mise bas ont été respectivement de 6,6 2 2,2, 10,3 $\pm 3,5$ et 18,4 $\pm 6,6 \mathrm{~kg}$ (tableau III). L'application des différents modèles d'analyse a mis en évidence l'existence de plusieurs sources de variation des caractères

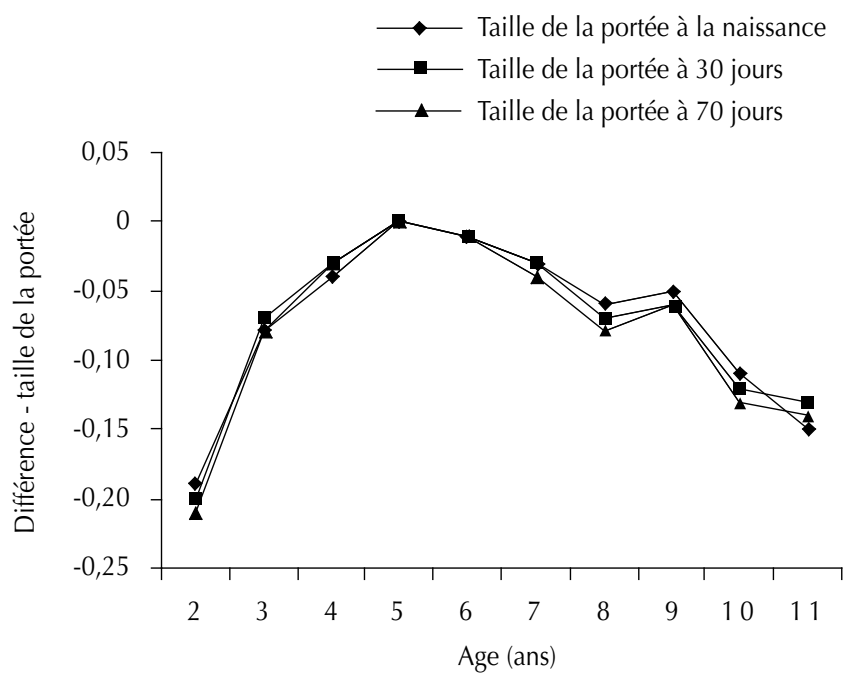

Figure 2 : effets de l'âge sur les tailles de la portée à la naissance, à 30 et à 70 jours après la mise bas de la brebis Noire de Thibar.

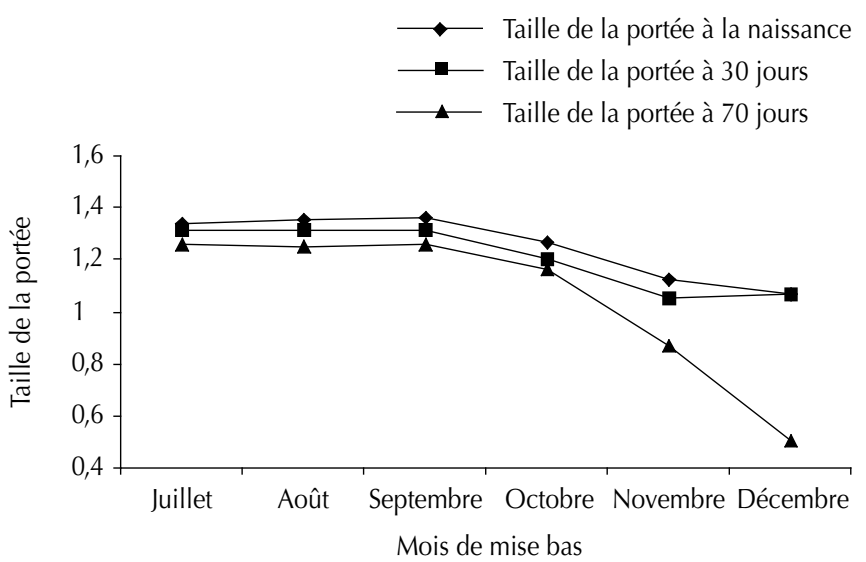

Figure 3 : effet du mois de mise bas sur les tailles de la portée à la naissance, à 30 et à 70 jours après la mise bas pour la brebis Noire de Thibar. de productivité pondérale de la brebis Noire de Thibar. Les coefficients de détermination des différents modèles ont varié entre 0,55 et 0,61 en fonction du paramètre étudié. La ferme, l'année de mise bas, le mois de mise bas, l'âge de la brebis et la taille de la portée à la naissance ont été des sources de variation importantes pour la productivité pondérale $(\mathrm{p}<0,001)$. Le facteur sexe de la portée a eu un effet significatif uniquement sur les poids de la portée à 30 et à 70 jours. A 70 jours, les poids des portées formées par des mâles seuls ont été plus élevés que ceux des portées de sexe mixte et composées de femelles seules. Comparées aux portées composées de femelles seules (prises comme référence), les différences de poids ont atteint à 70 jours après l'agnelage 0,6 et $1,0 \mathrm{~kg}$, respectivement pour les portées de sexe mixte et celles de sexe mâle. Les interactions entre la ferme et l'année de mise bas, entre l'année et le mois

\section{Tableau III}

Sources de variation des poids de la portée des brebis de race Noire de Thibar

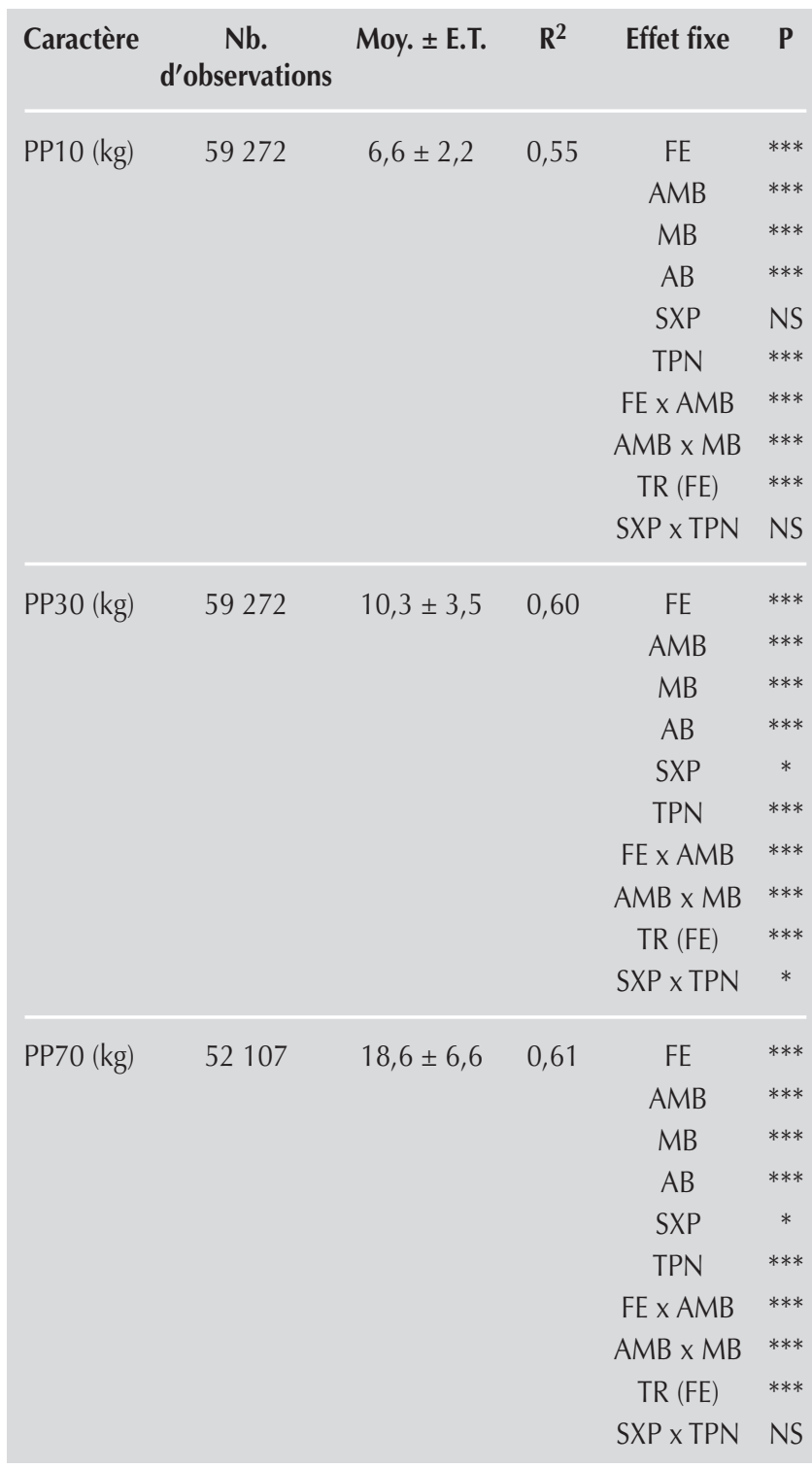

E.T. : écart-type ; FE : ferme ; AMB : année de mise bas ; MB : mois de mise bas ; $\mathrm{AB}$ : âge de la brebis ; SXP : sexe de la portée ; TPN : taille de la portée à la naissance ; TR : troupeau; PP10, PP30 et PP70 : poids de la portée respectivement à 10,30 et 70 jours après la mise bas

*** $\mathrm{P}<0,001 ; * \mathrm{P}<0,05 ; \mathrm{NS}:$ non significatif 
de mise bas, et le facteur troupeau intra-ferme ont également affecté de manière très significative tous les caractères étudiés. L'interaction sexe x taille de la portée n'a eu d'effet significatif $(\mathrm{p}<0,05)$ que pour le poids de la portée à 30 jours.

Les effets moyens de la taille de la portée à la naissance sur les poids de la portée à 10 et à 70 jours après la mise bas sont indiqués dans le tableau IV. Pour ces deux caractères de productivité, une nette supériorité des portées doubles et triples a été constatée par rapport aux portées simples. Cette supériorité s'est accrue au fur et à mesure de l'éloignement de la date de mise bas. Ainsi, les brebis ayant eu des portées doubles ou triples ont produit, 70 jours après l'agnelage, respectivement 8,7 et $12,5 \mathrm{~kg}$ de plus que les brebis ayant eu un seul agneau.

La figure 4 illustre l'effet net de l'âge de la brebis sur les poids de la portée. Les performances de productivité les plus élevées ont été observées dans la catégorie d'âge comprise entre 3 et 6 ans avec un maximum atteint à l'âge de 4 ans. Après 6 ans d'âge, les performances de productivité des brebis ont diminué. Les estimations des moindres carrés de l'interaction entre la taille et le sexe de la portée pour la productivité pondérale à 30 jours après la mise bas sont consignées dans la figure 5. Pour les portées composées d'un seul agneau, les mâles ont réalisé des performances plus élevées. Quand la portée a été composée de deux agneaux, les performances les plus élevées ont été attribuées au portées mixtes, suivies des mâles

\section{Tableau IV}

Estimation des moindres carrés des effets de la taille de la portée à la naissance sur les poids de la portée des brebis Noires de Thibar

$\begin{array}{lcc}\text { TPN } & \text { PP10 (kg) } & \text { PP70 (kg) } \\ \text { Simples } & -4,39 & -12,51 \\ \text { Doubles } & -1,36 & -3,84 \\ \text { Triples } & 0 & 0\end{array}$

TPN : taille de la portée à la naissance ; PP10 et PP70 : poids de la portée à 10 et 70 jours après la mise bas

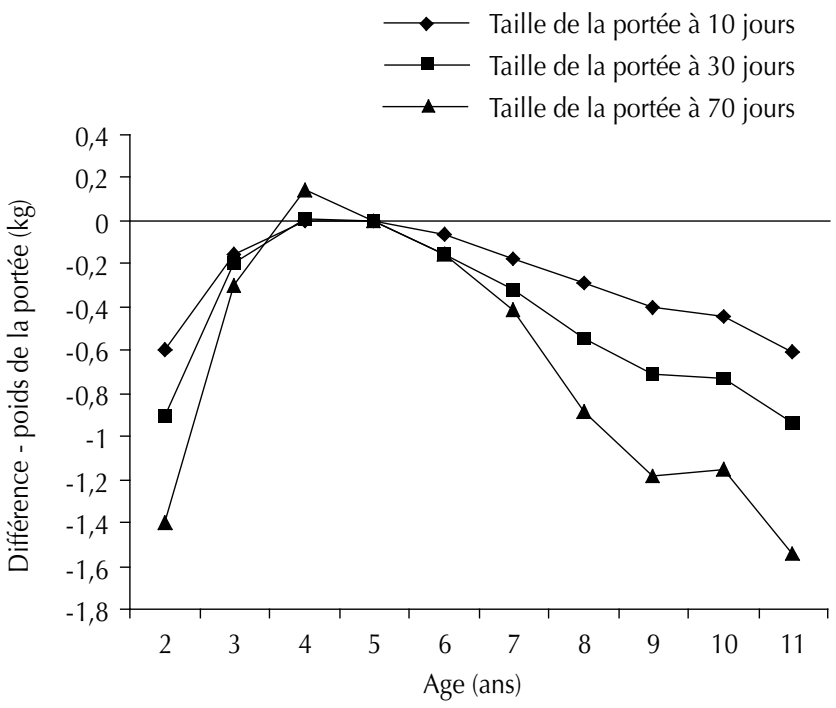

Figure 4 : effets de l'âge de la brebis Noire de Thibar sur les poids de la portée à 10, 30 et 70 jours après la mise bas. et enfin des portées formées uniquement de femelles. Les portées à trois agneaux, quel qu'ait été le nombre de mâles ou de femelles, ont eu des performances semblables.

\section{DISCUSSION}

\section{Paramètres de reproduction}

En comparant la Noire de Thibar aux autres races à viande en Tunisie, en l'occurrence la Barbarine et la Queue fine de l'Ouest d'après l'étude de Chafik (12), son taux de mise bas est apparu plus faible. Cependant, lorsque la comparaison entre les trois génotypes est restreinte au nord de la Tunisie (12), dans son berceau d'élevage, le taux de mise bas de la Noire de Thibar, avec une moyenne de 93,6 p. 100, est le plus élevé. A l'opposé, son taux de mise bas peut baisser à moins de 75 p. 100 dans le centre de la Tunisie où les conditions climatiques sont plus difficiles que dans le nord du pays. En ce qui concerne la taille de portée à la naissance, la moyenne obtenue dans cette étude pour les brebis de race Noire de Thibar a été supérieure à celles des deux autres races à viande tunisiennes. En effet, une taille de portée moyenne de 1,27 et de 1,13 est rapportée pour la Barbarine, respectivement par Ben Gara (4) et Jmal (20), et de 1,19 pour la Queue fine de l'Ouest par Lassoued et Rekik (25). Néanmoins, la race Noire de Thibar demeure moyennement prolifique comparée à d'autres races étrangères. Abdulkhaliq et coll. (1), Boujenane et coll. (8), et Long et coll. (26) rapportent des tailles de portée à la naissance respectivement de 1,49, 2,00 et 1,50 pour les races Columbia, D’Man et Suffolk.

Cette étude a montré un effet important de la ferme et de l'année aussi bien sur le taux de mise bas que sur la taille de la portée. Ce résultat classique qui illustre la dépendance des performances reproductives de l'environnement est aussi rapporté par d'autres auteurs pour d'autres races et dans d'autres régions du monde (10, 11, 30). Les fluctuations observées dans les paramètres de reproduction représentent, d'une part, le caractère aléatoire des variations climatiques au sud de la Méditerranée avec ses conséquences sur l'alimentation et l'état corporel des brebis. Ces mêmes fluctuations traduisent, d'autre part, des stratégies différentes de gestion

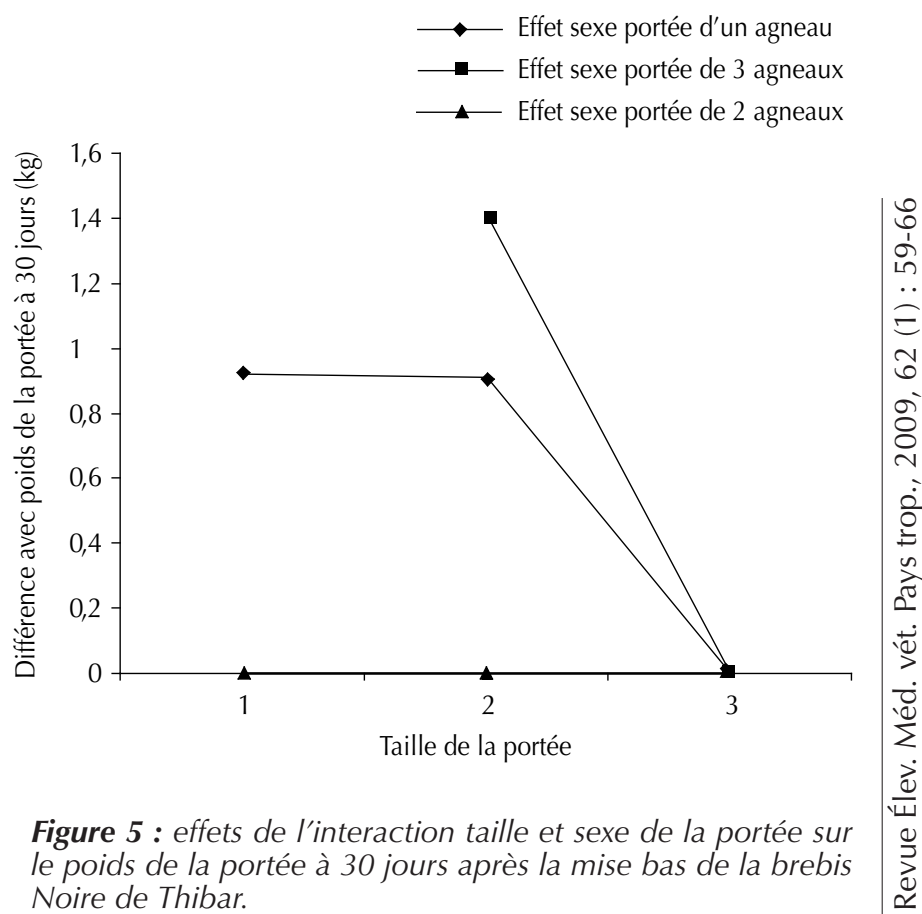


de l'alimentation et de la reproduction entre les exploitations (23). C'est la résultante de l'ensemble de ces facteurs de nature climatique et ayant trait à la conduite qui serait à la base des larges variations observées des paramètres de reproduction (28).

Par ailleurs, comme le rapportent Fogarty et coll. (17), les écarts entre les performances de productivité numérique enregistrés dans les fermes de la présente étude peuvent suggérer l'existence d'interactions génotype $\mathrm{x}$ environnement, non seulement entre régions géographiques, mais aussi entre des systèmes de production qui diffèrent dans une même région.

Il est également important de souligner l'effet de la saison d'agnelage sur les performances de productivité numérique. Cet effet a aussi été rapporté par Boujenane et coll. (9), Clément et coll. (14), et Niaré (30).

Dans l'analyse de la variable TPN, l'effet de la saison d'agnelage doit être interprété selon la période de fécondation (22). Ainsi, par exemple, les faibles valeurs de TPN des mois de novembre et de décembre correspondent à des accouplements de juin et juillet, période au cours de laquelle les températures élevées peuvent être préjudiciables à la qualité spermatique et à la survie embryonnaire. De plus, les travaux effectués par Lassoued et Khaldi (24) sur la race Noire de Thibar montrent qu'en juin et juillet le taux d'ovulation de la race est faible, ne dépassant pas 1,25. Pour les tailles de portée à 30 et à 70 jours après la mise bas, l'effet de la saison d'agnelage se manifeste à travers la survie des jeunes dans la portée (19).

\section{Productivité pondérale}

A 10,30 et 70 jours après la mise bas, les performances de productivité de la race Noire de Thibar les plus élevées ont été réalisées par les portées triples, composées uniquement de mâles et dont l'âge de la mère était de 4 à 5 ans. Par rapport à la Barbarine, la supériorité du poids de la portée de la Noire de Thibar à 70 jours après l'agnelage a été d'environ $1,4 \mathrm{~kg}$, bien que les performances des deux races à 30 jours soient sensiblement les mêmes $(10,3$ $\mathrm{kg}$ ) (20). Ce résultat confirme celui de Chalh (13), selon lequel les agneaux de la race Noire de Thibar ont une vitesse de croissance plus élevée entre 30 et 70 jours d'âge qu'au premier mois de vie. Ces poids de la portée aux âges types restent toutefois inférieurs à ceux rapportés par Aloulou et coll. (3) pour la race D'Man élevée en Tunisie qui est, par ailleurs, plus prolifique que la Noire de Thibar.

Les performances de productivité ont été très variables selon la ferme mais aussi selon le troupeau intra-ferme, un résultat corroboré par celui de Chalh (13) pour les caractères de croissance individuelle des agneaux de cette même race. Ces facteurs traduisent les effets de la localisation géographique, de la maîtrise de la conduite en général aux deux niveaux ferme et troupeau, et plus particulièrement du système alimentaire adopté. En effet, la Noire de Thibar est élevée au sein de systèmes de production régionaux variés (fermes privées, unités coopératives de production, sociétés de mise en valeur et de développement agricole, institutions étatiques), répondant chacun aux caractéristiques socio-économiques et climatiques locales, et adoptant des degrés d'intensification différents. On peut ajouter le facteur humain (surtout au niveau du troupeau), lié à la technicité des éleveurs, qui est sans doute déterminant.

L'influence de l'année de mise bas sur la productivité reflète la disponibilité des ressources alimentaires, elles-mêmes étroitement dépendantes de la pluviométrie. Cette variation se répercute sur la production laitière des brebis et, par conséquent, sur le développement et la croissance de leurs agneaux (27). Cependant, les fluctuations des performances sont d'autant plus importantes que l'on s'éloigne de la date de mise bas ; ceci peut être attribué au fait qu'aux premiers stades de vie, l'agneau demeure fortement dépendant de la production laitière de sa mère qui réagit aux fluctuations climatiques et nutritionnelles en mobilisant ses réserves corporelles. Cet effet maternel explique l'importance très significative de l'âge de la mère ; quand celle-ci est très jeune (2 ans) ou arrive en fin de leur carrière ( 9 ans et plus), la croissance de ses agneaux est plus faible. La tendance obtenue ici pour la Noire de Thibar a été similaire à celles rapportées par Abdulkhaliq et coll. (1), Boujenane et Bradford (7), et Bourfia et Touchberry (10), respectivement pour les races Columbia et D'Man.

\section{CONCLUSION}

Dans cette étude, qui valorise une très large base de données collectées dans des élevages intensifs, les performances de la race Noire de Thibar ont été modérées. Elles ne confirment pas les bonnes aptitudes zootechniques obtenues initialement dans les premiers noyaux de cette race établis dans les années 1960 (21), ni les résultats d'autres études qui s'étaient intéressées à un nombre très limité d'exploitations $(12,13)$.

Si, à l'échelle nationale, l'orientation était prise pour doter cette race d'un schéma d'amélioration génétique où la productivité des brebis était retenue dans un objectif de sélection, les résultats de cette étude représenteraient un pas important pour l'identification des sources de variation non génétiques qui affectent ce paramètre. Les variations observées traduisent probablement l'existence d'interactions génotype x système de production ; c'est cette variabilité qu'il faudrait cerner et exploiter dans les programmes d'amélioration de la race. Toutefois, l'absence d'un contrôle de paternité dans les élevages inscrits au contrôle des performances reste un obstacle important à la caractérisation génétique de la race, notamment à l'estimation des paramètres génétiques des caractères de productivité dont la connaissance demeure indispensable pour les prendre en compte dans un schéma de sélection. 
1. ABDULKHALIQ A.M., HARVEY W.R., PARKER C.F., 1989. Genetic parameters for ewe productivity traits in the Columbia, Suffolk and Targhee breeds. J. Anim. Sci., 69: 3250-3257.

2. ALOULOU R., 1990. Croissance pondérale de la race Barbarine dans le semi-aride Tunisien : coefficients de correction et paramètres génétiques. Mém. Cycle spécialisation, Institut national agronomique, Tunis, Tunisie, $59 \mathrm{p}$.

3. AlOULOU R., EL HENTATI H., REKIK M., BEN HAMOUDA M., 2004. Genetic parameters of weight productivity traits of D'Man ewes in Tunisia. In: 55th Annu. Meet. Eur. Assoc. Anim. Prod., Bled, Slovenia, 5-9 Sept. 2004.

4. BEN GARA A., 2000. Définition des objectifs de la sélection des ovins de race Barbarine en Tunisie. Options méditerr., Ser. A, 43 : 111-116.

5. BEN HAMOUDA M., 1985. Description biométrique et amélioration génétique de la croissance pondérale des ovins de race Barbarine. Thèse Doct., Université de Gand, Belgique, 166 p.

6. BEN HAMOUDA M. 2005. Minimum sequential records to adequately fit growth curve in Fat-Tailed Barbarine lambs and implication on genetic evaluation. In: Proc. 34th Biennial Session of ICAR, Sousse, Tunisia, 28 May - 3 June 2004, 6 p.

7. BOUJENANE I., BRADFORD G.E., 1991. Genetic effects on ewe productivity of crossing D'Man and Sardi breeds of sheep. J. Anim. Sci., 69: $525-530$

8. BOUJENANE I., BRADFORD G.E., FAMULA T.R., 1991. Inheritance of litter size and its components in crosses between the D'Man and Sardi breeds of sheep. J. Anim. Sci., 69: 517-524.

9. BOUJENANE I., CISSE M.F., KANSARI J., HAZZAM R., 2002. Sheep productivity in autumn and spring lambing from three cross breeding systems. In: VIIth World Congr. Genetics applied to livestock production, Montpellier, France, 19-23 Aug. 2002.

10. BOURFIA M., TOUCHBERRY R.W., 1993. Diallel cross of three Moroccan breeds of sheep. II. Reproductive performance and productivity of purebred ewe. J. Anim. Sci., 71: 882-887.

11. BUNGE R., THOMAS D.L., STOOKEY J.M., 1990. Factors affecting productivity of Rambouillet ewes mated to ram lambs. J. Anim. Sci., 68: 2253-2262.

12. CHAFIK A., 2003. Comparaison des performances de production et de reproduction des races ovines à viande tunisiennes. Mém. Diplôme Etudes approfondies, Institut national agronomique, Tunis, Tunisie, 115 p.

13. CHALH A., 1996. Caractérisation génétique de la race ovine tunisienne "Noire de Thibar » sur la base des caractères pondéraux et approche $d^{\prime}$ indexation des futurs reproducteurs pour une meilleure croissance. Thèse Doct., faculté des Sciences, Tunis, Tunisie, 162 p.

14. CLEMENT V., POIVEY J.P., FAUGERE O., TILLARD E., LANCELOT R., GUEYE A., RICHARD D., BIBE B., 1997. Etude de la variabilité des caractères de reproduction chez les petits ruminants en milieu d'élevage traditionnel au Sénégal. Revue. Elev. Méd. vét. Pays. trop., 50 : 235-249.

15. CROSTON D., 1995. Breeding strategy for the British sheep industry. Stratégies pour I'amélioration génétique des ovins et caprins. Options méditerr., Ser A, 11 : 203-210.

16. DOYLE E.W., MORRICAL D.G., 1991. The National Sheep Improvement Program: A review. J. Anim. Sci., 69: 3872-3881.

17. FOGARTY N.M., DICKERSON G.E., YOUNG L.D., 1985. Lamb production and its components in pure breeds and composite lines. III. Genetic parameters. J. Anim. Sci., 66: 40-57.
18. GABINA D., 1995. Amélioration génétique des ovins à viande. L'élevage ovin en zones arides et semi-arides. Options méditerr., Ser. A, $6: 87-99$

19. GAMA L.T., DICKERSON G.E., YOUNG L.D., LEYMASTER K.A. 1991. Genetic and phenotypic variation in sources of preweaning lamb mortality. J. Anim. Sci., 69: 2744-2753.

20. JMAL S., 1995. Paramètres génétiques des caractères de la productivité de la brebis Barbarine dans le semi-aride Tunisien. Mém. Cycle spécialisation, Institut national agronomique, Tunis, Tunisie, 84 p.

21. KALLEL A., 1968. Le mouton Noir de Thibar. Thèse Doct. vét., faculté de Médecine et de Pharmacie, Toulouse, France.

22. KHALDI G., 1984. Variations saisonnières de l'activité ovarienne, du comportement de l'œstrus et de la durée de l'anœstrus post partum des femelles ovines de race Barbarine. Influence du niveau alimentaire et la présence du mâle. Thèse Doct., université de Montpellier, France, 168 p.

23. LASSOUED N., ATTI N., 1996. Maîtrise des techniques d'élevage ovin en conditions difficiles. Tunis, Tunisie, secrétariat à la Recherche scientifique et à la Technologie, $16 \mathrm{p}$.

24. LASSOUED N., KHALDI G., 1995. Variation saisonnières de l'activité sexuelle des brebis de race Queue fine de l'Ouest et Noire de Thibar. L'élevage ovin en zones arides et semi-arides. Options méditerr., Ser. A, $6: 27-34$

25. LASSOUED N., REKIK M., 2001. Differences in reproductive efficiency between female sheep of the Queue fine de I'Ouest purebred and their first cross with D'Man. Anim. Res., 50: 373-381.

26. LONG E.T., THOMAS D.L., FERNANDO R.L., LEWIS J.M., GARRIGUS U.S., WALDRON D.F., 1989. Estimation of individual and maternal heterosis, repeatability and heritability for ewe productivity and its components in Suffolk and Targhee sheep. J. Anim. Sci.; 67: 12081217

27. MARIA G., GABINA D., ARRANZ J., URARTE E., 1991. Factors of variation and correlation coefficients of production and composition criteria of milk from Latxa ewes. Invest. Agrar., Prod. Sanid. Anim., 6: 189-198.

28. MARTIN G.B., MILTON G.T.B., DAVIDSON R.H., BANCHERO HUNZICKER G.E., LINDSAY D.R., BLACHE D., 2004. Natural methods for increasing reproductive efficiency in small ruminants. Anim. Reprod. SCi., 82-83: 231-246.

29. MINISTERE DE L'AGRICULTURE DE TUNISIE, 1995. Enquête sur les structures des exploitations agricoles en Tunisie. Tunis, Tunisie, direction générale de la Planification, du Développement et des Investissements agricoles

30. NIARE T., 1995. Croissance présevrage des agneaux et productivité en milieu traditionnel soudano-sahélien au Mali. Revue Elev. Méd. vét Pays. trop., 48 : 195-202

31. PARKER C.F., POPE A.L., 1983. The US sheep industry: Changes and challenges. J. Anim. Sci., 57 (Suppl.2): 75.

32. REKIK M., ALOULOU R., BEN HAMOUDA M., 2006. Small ruminants breeds of Tunisia. In: Iniguez L. Ed., Characterisation of small ruminant breeds in West Asia and North Africa. Vol. 2: North Africa. Aleppo, Syria, ICARD, p. 91-140.

33. SAS, 1989. Users' Guide: Statistics, Vers. 6. Cary, N.C., USA, SAS Institute.

Accepté le 09.02.2010 


\section{Summary}

Ben Salem I., Rekik M., Hammami H., Ben Hamouda M., Aloulou R., Sâadoun L. Non-Genetic Factors of Variation of the Productivity of the Noire de Thibar Ewe

An initial database containing growth records of 101,000 lambs obtained within the context of the national meat sheep improvement program was used to quantify reproductive and productivity (total litter weight) traits of ewes of the Noire de Thibar breed, and to determine the influence of non-genetic factors on these traits. A database was then generated: it comprised 64,137 lambings over a 12-year period (1991-2002), and 25 farms and 48 flocks. The mean fertility rate in the flocks was $80.1 \pm 12.9 \%$ with highly significant effects $(p<0.001)$ of the farm and lambing year. At birth, the average litter size per lambing ewe was $1.32 \pm 0.47$. It dropped to $1.23 \pm 0.55$ at 70 days after lambing. All main sources of variation (farm, lambing year, lambing month, and dam age) retained in the model as well as their interactions significantly affected the litter size at different stages after lambing. Survival of all the lambs in the litters between birth and 70 days post-lambing was observed in $89.9 \%$ of the ewes. Entire loss of the litter was observed in only $5.8 \%$ of the ewes. An analysis of variance revealed that factors such as the lambing year, lambing month, dam age, sex of the litter, and litter size at birth had a highly significant effect on the proportions of ewes with different levels of lambs' survival within litters. On average, the productivity of the Noire de Thibar was $6.6 \pm 2.2,10.3 \pm 3.5$ and $18.6 \pm 6.6$ $\mathrm{kg}$ at 10, 30 and 70 days after lambing, respectively. The main factors of variation (farm, lambing year, lambing month, dam age, litter size), and some second-level interactions had highly significant effects $(p<0.001)$ on the studied productivity traits. Ewes aged four years and which had male triplets yielded the highest productivities at 70 days after lambing. This preliminary work on the reproductive and productivity traits of Noire de Thibar ewes is essential to develop a ewe selection index as part of a genetic improvement scheme for this breed.

Keywords: Sheep - Ewe - Birth rate - Litter size - Viability Productivity - Tunisia.

\section{Resumen}

Ben Salem I., Rekik M., Hammami H., Ben Hamouda M., Aloulou R., Sâadoun L. Factores de variación no genética de la productividad de las ovejas de raza Negra de Thibar

Con el fin de medir los rendimientos reproductivos y la productividad (peso total de la camada) de ovejas Negras de Thibar y de determinar la influencia de los factores no genéticos sobre estos parámetros, se utilizó una base de datos inicial constituida de 101000 corderos controlados para el crecimiento dentro del cuadro del programa nacional de mejoramiento de ovinos de carne. Seguidamente se generó una base de datos ajustada por oveja, con un total de 64137 partos representando 12 años (1991-2002), 25 fincas y 48 hatos. La tasa de fertilidad media de los hatos de la raza Negra de Thibar fue de 80,1 $\pm 12,9 \%$ con efectos importantes $(p<0,001)$ de la finca y del año de parto. Al nacimiento, el tamaño de la camada promedio por oveja parida alcanzó 1,32 \pm 0,47; ésta bajó a 1,23 $\pm 0,55$ a 70 días después del parto. Todas las principales fuentes de variación retenidas en el modelo (finca, año de parto, mes de parto y edad de la oveja), así como sus interacciones afectaron significativamente el tamaño de la camada en los diferentes estadios post del parto. La sobrevida de los corderos por camada entre el nacimiento y los 70 días fue observada en $89.9 \%$ de las ovejas y solamente $5,8 \%$ de las ovejas perdieron la totalidad de los corderos de la camada. Un análisis de varianza reveló que los factores año de parto, mes de parto, edad de la oveja, sexo de la camada y tamaño de la camada, tuvieron un efecto muy significativo sobre la proporción de ovejas que presentan diferentes niveles de sobrevida de los corderos en el seno de la camada. En promedio, la productividad de la oveja Negra de Thibar a 10, 30 y 70 días post parto fue respectivamente de $6,6 \pm 2,2,10,3 \pm 3,5$ y 18,6 $\pm 6,6 \mathrm{~kg}$. Las principales fuentes de variación (finca, año de parto, mes de parto, edad de la oveja y tamaño de la camada), así como ciertas interacciones de segundo nivel tuvieron efectos significativos $(p<0,001)$ sobre los caracteres de productividad estudiados. La categoría de las ovejas de cuatro años con camadas de trillizos, todos machos, se asoció a los niveles de productividad más elevados a 70 días. El presente estudio preliminar sobre los caracteres reproductivos y de productividad de la oveja Negra de Thibar es esencial para la elaboración de un índice de selección de hembras que podrá ser incorporado en un eventual esquema de mejoramiento genético de esta raza.

Palabras clave: Ovino - Oveja - Tasa de natalidad - Tamaño de la camada - Supervivencia - Productividad - Túnez. 